\title{
AN UNBOUNDED OPERATOR WITH SPECTRUM IN A STRIP AND MATRIX DIFFERENTIAL OPERATORS
}

\author{
MICHAEL GIL'
}

(Received 16 February 2021; accepted 1 March 2021; first published online 16 April 2021)

\begin{abstract}
Let $A$ and $\tilde{A}$ be unbounded linear operators on a Hilbert space. We consider the following problem. Let the spectrum of $A$ lie in some horizontal strip. In which strip does the spectrum of $\tilde{A}$ lie, if $A$ and $\tilde{A}$ are sufficiently 'close'? We derive a sharp bound for the strip containing the spectrum of $\tilde{A}$, assuming that $\tilde{A}-A$ is a bounded operator and $A$ has a bounded Hermitian component. We also discuss applications of our results to regular matrix differential operators.
\end{abstract}

2020 Mathematics subject classification: primary 47A10; secondary 47A55, 47B10, $47 \mathrm{E} 05$.

Keywords and phrases: differential operator, Hilbert space, spectrum localisation.

\section{Introduction and statement of the main result}

Let $\mathcal{H}$ be a complex separable Hilbert space with a scalar product $(\cdot, \cdot)$, norm given by $\|\cdot\|=\sqrt{(\cdot, \cdot)}$ and unit operator $I$. By $\mathcal{L}(\mathcal{H})$ we denote the set of all bounded operators in $\mathcal{H}$. For an operator $A$ on $\mathcal{H}, D(A)$ is its domain, $A^{*}$ and $A^{-1}$ are the adjoint and inverse operators, respectively, $\sigma(A)$ is the spectrum, $R_{z}(A)=(A-z I)^{-1}(z \notin \sigma(A))$ is the resolvent, and $\lambda_{j}(A)(j=1,2, \ldots)$ denote the eigenvalues of $A$ taken with their multiplicities. In addition, for $\omega>0$, we denote by

$$
H_{\omega}:=\{z \in \mathbb{C}:|\operatorname{Im} z|<\omega\}
$$

the horizontal strip of height $2 \omega$ which is symmetric with respect to the real axis. Following [10, Section 4.1], we will say that an operator $A$ on $\mathcal{H}$ is a strip-type operator of height $\omega$ (in short, $A \in \operatorname{Strip}(\omega)$ ) if $\sigma(A) \subset H_{\omega}$ and $\sup _{|\operatorname{Im} z| \geq \omega^{\prime}}\left\|R_{z}(A)\right\|<\infty$ for all $\omega^{\prime}>\omega$. Finally,

$$
\omega_{\mathrm{st}}(A):=\inf \{\omega \geq 0: A \in \operatorname{Strip}(\omega)\}
$$

is called the spectral height of $A$.

We consider the following problem. Let $A$ and $\tilde{A}$ be strip-type operators on $\mathcal{H}$. In which strip does the spectrum of $\tilde{A}$ lie if $\omega_{\text {st }}(A)$ is known and $\tilde{A}$ and $A$ are sufficiently 'close'? We also discuss applications of our results to matrix differential operators.

(C) Australian Mathematical Publishing Association Inc. 2021. This is an Open Access article, distributed under the terms of the Creative Commons Attribution licence (http://creativecommons.org/ licenses/by/4.0/), which permits unrestricted re-use, distribution, and reproduction in any medium, provided the original work is properly cited. 
The strip-type operators form a wide class of unbounded operators in a Banach space. The important example here is the logarithm of a sectorial operator, arising in various applications (see [10,16]). The natural functional calculus for strip-type operators appears first in [2]. It is discussed in [11] in a general setting and used in [3]. The theory of strip-type operators is developed in [9, 16, 17] and the references given therein. For more details, see [10, Ch. 4]. To the best of our knowledge, the above-mentioned problem has not been considered in the literature, although it is important for the localisation of spectra and in various applications.

Furthermore, $A$ is said to be a strong strip-type operator of height $\omega$, if for any $\omega^{\prime}>\omega$ there is an $L_{\omega^{\prime}}$ such that

$$
\left\|R_{z}(A)\right\| \leq \frac{L_{\omega^{\prime}}}{|\operatorname{Im} z|-\omega^{\prime}} \quad \text { for }|\operatorname{Im} z|>\omega^{\prime} .
$$

From [10, Example 4.1.1.2, page 92], if $i A$ generates a $C_{0}$-group $e^{i A t}$ in a Hilbert space, then $A$ is a strong strip-type operator of height $\theta\left(e^{i A t}\right)$, where $\theta\left(e^{i A t}\right)$ is the group type of $e^{i A t}$. In particular,

$$
\omega_{\mathrm{st}}(A)=\theta\left(e^{i A t}\right)
$$

Throughout the paper it is assumed that $D(A)$ is dense in $\mathcal{H}, A=A_{R}+i A_{I}$, where $A_{R}$ and $A_{I}$ are self-adjoint operators, and

$$
A_{I} \in \mathcal{L}(\mathcal{H})
$$

According to the Stone theorem (see [10, Section 4.1]), the operator $i A_{R}$ generates a $C_{0}$-group $e^{i t A_{R}}(-\infty<t<\infty)$ of unitary operators. In particular, for $t \geq 0$ it is a semigroup. Moreover, by [5, Theorem II.4.6], $i A_{R}$ generates a bounded analytic semigroup. Hence, by [5, Proposition III.1.12], iA generates a bounded analytic semigroup, since $A_{I}$ is bounded. Thus, under condition (1.2), $A$ is a strip-type operator and therefore (1.1) holds.

Let

$$
D(\tilde{A})=D(A) \quad \text { and } \quad q:=\|A-\tilde{A}\|<\infty .
$$

Then $\left\|\tilde{A}_{I}\right\| \leq q+\left\|A_{I}\right\|$ and therefore $\tilde{A}$ is also a strip-type operator.

We introduce the notation $x(t)=e^{i t A} x_{0} \quad\left(x_{0} \in D(A)\right), \quad \alpha\left(A_{I}\right)=\sup \sigma\left(A_{I}\right)$ and $\beta\left(A_{I}\right)=\inf \sigma\left(A_{I}\right)$. Then

$$
\frac{d}{d t}(x(t), x(t))=2 \operatorname{Re}(i A x(t), x(t))=-2\left(A_{I} x, x\right) \leq-2 \beta\left(A_{I}\right) \leq 2\left\|A_{I}\right\|\|x(t)\|^{2}
$$

and

$$
\frac{d}{d t}(x(t), x(t))=-2\left(A_{I} x, x\right) \geq-2 \alpha\left(A_{I}\right)\left(A_{I} x, x\right) .
$$


Consequently, $\left\|e^{i A t} x_{0}\right\| \leq\left\|x_{0}\right\| e^{\left\|A_{I}\right\| t}$ for $\left.t \geq 0\right)$. Thus, from $(1.1), \quad \omega_{\mathrm{st}}(A) \leq\left\|A_{I}\right\|$. Similarly,

$$
\omega_{\mathrm{st}}(\tilde{A}) \leq\left\|\tilde{A}_{I}\right\|
$$

This inequality is rather rough. Below, we present a considerably sharper estimate.

To this end, note that according to (1.1), $\left\|e^{ \pm i A t}\right\| \leq$ const. $e^{\omega_{\mathrm{st}} t}(t \geq 0)$, and thus the operators $-(c I \pm i A)$, for $c \in \mathbb{R}$, generate the exponentially stable semigroups $e^{-(c I \pm i A) t}$, provided $c>\omega_{\mathrm{st}}$. Hence, the integral

$$
X_{c}:=\int_{0}^{\infty} e^{-(i A+c I)^{*} t} e^{-(i A+c I) t} d t \quad\left(c>\omega_{\mathrm{st}}\right)
$$

strongly converges and

$$
\left\|X_{c}\right\| \leq \int_{0}^{\infty} e^{-2 c t}\left\|e^{-i A t}\right\|^{2} d t
$$

We are now in a position to formulate our main result, which we prove in Section 2.

THEOREM 1.1. Let conditions (1.2) and (1.3) hold. Let $X_{c}$ be defined by (1.5) for some $c>\omega_{\mathrm{st}}$. Then $\omega_{\mathrm{st}}(\tilde{A})<c$, provided $q\left\|X_{c}\right\|<1 / 2$.

Now put

$$
w_{c}(A):=\frac{1}{2 \pi} \int_{-\infty}^{\infty}\left\|(i A+(i s+c) I)^{-1}\right\|^{2} d s .
$$

By the classical Parseval-Plancherel equality [1, Theorem 5.2.1], for any $x \in \mathcal{H}$,

$$
\begin{aligned}
\left(X_{c} x, x\right)=\left(\int_{0}^{\infty} e^{-(I c+i A)^{*} t} e^{-(I c+i A) t} x d t, x\right) & =\int_{0}^{\infty}\left\|e^{-(A i+I c) t} x\right\|^{2} d t \\
& =\frac{1}{2 \pi} \int_{-\infty}^{\infty}\left\|(i A+(i s+c) I)^{-1} x\right\|^{2} d s .
\end{aligned}
$$

Hence,

$$
\left\|X_{c}\right\| \leq w_{c}(A)
$$

If $A$ is normal, that is, $A A^{*}=A^{*} A$, then by the spectral representation (see, for instance, [12]), we easily see that $\left\|e^{i A t}\right\|=e^{-t \beta(A)}$, where $\beta(A):=\inf \operatorname{Im} \sigma(A)$ and $t \geq 0$. But $\beta(A) \geq-\omega_{\text {st }}(A)$. Therefore,

$$
\left\|X_{c}\right\| \leq \int_{0}^{\infty} e^{-2(c+\beta(A))} d t=\frac{1}{2(c+\beta(A))}=\frac{1}{2\left(c-\omega_{\mathrm{st}}(A)\right)} \quad\left(c>\omega_{\mathrm{st}}(A)\right) .
$$

Making use of Theorem 1.1, we obtain $\omega_{\text {st }}(\tilde{A}) \leq \omega_{\text {st }}(A)+q+\epsilon$ for $\epsilon>0$. Hence, letting $\epsilon \rightarrow 0$, we arrive at the following result.

Corollary 1.2. Let conditions (1.2) and (1.3) hold and let $A$ be normal. Then $\omega_{\mathrm{st}}(\tilde{A}) \leq \omega_{\mathrm{st}}(A)+q$. In particular, if $A$ is self-adjoint, then $\omega_{\mathrm{st}}(\tilde{A}) \leq q$. 
Let us show that Theorem 1.1 is sharp. To this end, assume that $K \in \mathcal{L}(\mathcal{H})$ and $A$ are self-adjoint commuting operators and $\tilde{A}=A+i K$. Suppose also that $\sigma(A)$ and $\sigma(K)$ are discrete. Then $\sigma(\tilde{A})$ consists of the eigenvalues

$$
\lambda_{j k}(\tilde{A})=\lambda_{j}(A)+i \lambda_{k}(K) \quad(j, k=1,2, \ldots) .
$$

Hence, $\omega_{\text {st }}(\tilde{A})=\sup _{k}\left|\lambda_{k}(K)\right|=q$, since $q=\|\tilde{A}-A\|=\|K\|=\sup _{k}\left|\lambda_{k}(K)\right|$. But due to Corollary $1.2, \omega_{\mathrm{st}}(\tilde{A}) \leq q$, since $\omega_{\mathrm{st}}(A)=0$. So the bound in Theorem 1.1 is attained in this case.

\section{Proof of Theorem 1.1}

We need the following well-known theorem (see [4, Theorem 5.1.3, page 217]).

THEOREM 2.1. Suppose that B is the infinitesimal generator of the $C_{0}$-semigroup $T(t)$ on a Hilbert space $\mathcal{H}$. Then $T(t)$ is exponentially stable if and only if there exists a bounded positive definite operator $P$ such that

$$
(B z, P z)+(P z, B z)=-(z, z) \quad(z \in D(B)) .
$$

Moreover, if $B$ is the infinitesimal generator of an exponentially stable $C_{0}$-semigroup then from [4, Section 5.5.3a, Equation (5.62)], for any $Q \in \mathcal{L}(\mathcal{H})$ the equation

$$
\left(B z_{1}, P z_{2}\right)+\left(P z_{1}, B z_{2}\right)=-\left(z_{1}, Q z_{2}\right)
$$

has a solution $P \in \mathcal{L}(\mathcal{H})$ which, again by [4, Section to 5.5.3a], is representable as

$$
P=\int_{0}^{\infty} e^{B^{*} t} Q e^{B t} d t .
$$

For a self-adjoint operator $S$ we write $S>0(S<0)$, if $S$ is positive (negative) definite. Let $D(B)=D\left(B^{*}\right)$ and $B^{*} P+P B=-C^{2}$ (with $C>0$ ) on $D(B)$ for some positive definite $P \in \mathcal{L}(\mathcal{H})$. Then

$$
C^{-1} B^{*} P C^{-1}+C^{-1} P B C^{-1}=C^{-1} B^{*} C C^{-1} P C^{-1}+C^{-1} P C^{-1} C B C^{-1}=-I .
$$

That is, $M^{*} Y+Y M=-I$, where $M=C B C^{-1}$ and $Y=C^{-1} P C^{-1}$.

According to Theorem 2.1, $M$ generates an exponentially stable semigroup. Since $M$ and $B$ are similar, we arrive at the following result.

Corollary 2.2. Let $D(B)=D\left(B^{*}\right)$ and $B^{*} P+P B<0$ on $D(B)$ for some positive definite $P \in \mathcal{L}(\mathcal{H})$. Then $\sup \operatorname{Re} \sigma(B)<0$.

Proof of TheOREM 1.1. From (2.3),

$$
(c I+i A)^{*} X_{c}+X_{c}(c I+i A)=I .
$$

Put $E=\tilde{A}-A$. Then from (2.4),

$$
\begin{aligned}
(i \tilde{A}+c I)^{*} X_{c}+X_{c}(i \tilde{A}+c I) & =(i A+c I)^{*} X_{c}+X_{c}(i A+c I)-i E^{*} X_{c}+i X_{c} E \\
& =I-i E^{*} X_{c}+i X_{c} E .
\end{aligned}
$$


If $2 q\left\|X_{c}\right\|<1$, then $(i \tilde{A}+c I)^{*} X_{c}+X_{c}(i \tilde{A}+c I)>0$. By Corollary 2.2, it follows that sup $\operatorname{Re} \sigma(-i \tilde{A}-c I)<0$. So $-c-\operatorname{Re}(i x-y)=-c+y<0$ for any $x+i y \in \sigma(\tilde{A})$. Thus sup $\operatorname{Im} \sigma(\tilde{A})<c$. Replacing $\tilde{A}$ by $-\tilde{A}$ and proceeding in the same way, we find $-c+\operatorname{Re}(i x-y)=-c-y<0$. Thus $\inf \operatorname{Im} \sigma(\tilde{A})>-c$. This proves the theorem.

\section{Spectral strips of differential operators with matrix coefficients}

Let $L^{2}=L^{2}\left([0,1], \mathbb{C}^{n}\right)$ be the space of functions defined on $[0,1]$ with values in $\mathbb{C}^{n}$ and the scalar product

$$
(f, h)_{L^{2}}=\int_{0}^{1}(f(x), h(x))_{n} d x \quad\left(f, h \in L^{2}\right),
$$

where $(\cdot, \cdot)_{n}$ means the scalar product in $\mathbb{C}^{n}$. On the domain

$$
D(A)=\left\{u \in L^{2}: u^{\prime \prime} \in L^{2} \text { and } u(0)=u(1)=0\right\},
$$

consider the operator

$$
\tilde{A}=-\frac{d^{2}}{d x^{2}}+C(x) \quad(x \in(0,1)),
$$

where $C(x)$ is an $n \times n$ matrix continuously dependent on $x$. We consider this operator as a perturbation of the operator

$$
A=-\frac{d^{2}}{d x^{2}}+C_{0} \quad(x \in(0,1))
$$

with a constant $n \times n$ matrix $C_{0}$. By way of example, one can take $C_{0}=C(0)$ or $C_{0}=\int_{0}^{1} C(x) d x$.

Clearly,

$$
\left(A_{I} f\right)(x)=C_{0 I} f(x) \quad\left(f \in L^{2}, x \in[0,1], C_{0 I}=\left(C_{0}-C_{0}^{*}\right) / 2 i\right)
$$

and

$$
q=\|A-\tilde{A}\|_{L^{2}} \leq \sup _{x}\left\|C(x)-C_{0}\right\|_{n} .
$$

Here $\|A-\tilde{A}\|_{L^{2}}$ is the operator norm in $L^{2}$ of $A-\tilde{A}$ and $\|\cdot\|_{n}$ means the spectral matrix norm (the operator norm with respect to the Euclidean vector norm).

Take into account that the operator $S$ defined on $D(A)$ by $S:=-d^{2} / d x^{2}$ commutes with constant matrices. Since the eigenvalues of $S$ are $\pi^{2} k^{2}(k=1,2, \ldots)$, by simple calculations we can show that $\sigma(A)$ consists of the eigenvalues $\lambda_{j k}(A)=\pi^{2} k^{2}+\lambda_{j}\left(C_{0}\right)$ $(k=1,2, \ldots, j=1, \ldots, n)$, where $\lambda_{j}\left(C_{0}\right)$ are the eigenvalues of $C_{0}$ taken with their multiplicities. Thus,

$$
\omega_{\mathrm{st}}(A)=\omega_{\mathrm{st}}\left(C_{0}\right):=\max _{j}\left|\operatorname{Im} \lambda_{j}\left(C_{0}\right)\right|
$$


Since $S$ and $C_{0}$ commute, we have $e^{i A t}=e^{i C_{0} t} e^{i S t}$. Hence, taking into account that $S=$ $S^{*}$ and therefore $\left\|e^{i S t}\right\|=1$, we can write $\left\|e^{i A t}\right\|_{L^{2}} \leq\left\|e^{i C_{0} t}\right\|_{n}$ and

$$
\left\|X_{c}\right\|_{L^{2}} \leq \int_{0}^{\infty} e^{-2 c t}\left\|e^{-i C_{0} t}\right\|_{n}^{2} d t .
$$

To estimate $\left\|e^{i C_{0} t}\right\|_{n}$, for an $n \times n$ matrix $M$, introduce the quantity $g(M)$ which measures the departure from normality:

$$
g(M):=\left[N_{2}^{2}(M)-\sum_{k=1}^{n}\left|\lambda_{k}(M)\right|^{2}\right]^{1 / 2},
$$

where $N_{2}(M):=\left(\operatorname{trace}\left(M^{*} M\right)\right)^{1 / 2}$ is the Hilbert-Schmidt (Frobenius) norm of $M$ and $\lambda_{k}(M)(k=1, \ldots, n)$ are the eigenvalues of $M$ taken with their multiplicities.

Various properties of $g(M)$ can be found in [8, Section 3.1]. In particular,

$$
g^{2}(M) \leq N_{2}^{2}(M)-\mid \text { trace } M^{2} \mid
$$

and

$$
g^{2}(M) \leq 2 N_{2}^{2}\left(M_{I}\right) \quad\left(\text { where } M_{I}=\left(M-M^{*}\right) / 2 i\right) .
$$

In addition, $g(z M)=|z| g(M)$ for $z \in \mathbb{C}$. If $M$ is a normal matrix, that is, $M M^{*}=M^{*} M$, then $g(M)=0$. By [8, Theorem 3.5], for any $n \times n$ matrix $M$,

$$
\left\|e^{M t}\right\| \leq \exp [\alpha(M) t] \sum_{k=0}^{n-1} \frac{g^{k}(M) t^{k}}{(k !)^{3 / 2}} \quad\left(\alpha(M)=\max _{k} \operatorname{Re} \lambda_{k}(M), t \geq 0\right) .
$$

But $\alpha\left(i C_{0}\right) \leq \omega_{\mathrm{st}}\left(C_{0}\right)$ and $g\left(i C_{0}\right)=g\left(C_{0}\right)$. Thus,

$$
\left\|e^{i C_{0} t}\right\| \leq \exp \left[\omega_{\mathrm{st}}\left(C_{0}\right) t\right] \sum_{k=0}^{n-1} \frac{g^{k}\left(C_{0}\right) t^{k}}{(k !)^{3 / 2}} \quad(t \geq 0)
$$

and from (3.3),

$$
\begin{aligned}
\left\|X_{c}\right\|_{L^{2}} & \leq \int_{0}^{\infty} \exp \left[-2\left(c-\omega_{\mathrm{st}}\left(C_{0}\right)\right) t\right]\left(\sum_{k=0}^{n-1} \frac{g^{k}\left(C_{0}\right) t^{k}}{(k !)^{3 / 2}}\right)^{2} d t \\
& =\int_{0}^{\infty} \exp \left[-2\left(c-\omega_{\mathrm{st}}\left(C_{0}\right)\right) t\right] \sum_{j, k=0}^{n-1} \frac{g^{j+k}\left(C_{0}\right) t^{k+j}}{(j ! k !)^{3 / 2}} d t \quad\left(c>\omega_{\mathrm{st}}\left(C_{0}\right)\right) .
\end{aligned}
$$

Since

$$
\int_{0}^{\infty} \exp [-s t] t^{k} d t=\frac{k !}{s^{k+1}} \quad(s>0),
$$

we find $\left\|X_{c}\right\| \leq \frac{1}{2} \zeta\left(c-\omega_{\text {st }}\left(C_{0}\right)\right)$, where

$$
\zeta(s)=\sum_{j, k=0}^{n-1} \frac{(j+k) ! g^{j+k}\left(C_{0}\right)}{2^{j+k} s^{k+j+1}(j ! k !)^{3 / 2}} \quad(s>0) .
$$


Now Theorem 1.1 implies the following result.

COROLlary 3.1. Let $\tilde{A}$ be defined by (3.1) and, for some $c>\omega_{\mathrm{st}}\left(C_{0}\right)$, let the condition

$$
q \zeta\left(c-\omega_{\mathrm{st}}\left(C_{0}\right)\right)<1
$$

hold. Then $\omega_{\mathrm{st}}(\tilde{A})<c$.

Let $x_{n}$ be the unique nonnegative root of the equation

$$
q \zeta(y)=q \sum_{j, k=0}^{n-1} \frac{(j+k) ! g^{j+k}\left(C_{0}\right)}{2^{j+k} y^{k+j+1}(j ! k !)^{3 / 2}}=1 \quad(y>0),
$$

which is equivalent to the equation

$$
y^{2 n}=q \sum_{j, k=0}^{n-1} \frac{(j+k) ! g^{j+k}\left(C_{0}\right)}{2^{j+k}(j ! k !)^{3 / 2}} y^{2 n-k-j-1}=1 .
$$

If $y>x_{n}+\omega_{\mathrm{st}}\left(C_{0}\right)$, then $q \zeta(y)<q \zeta\left(x_{n}\right)=1$. Now Corollary 3.1 implies $\omega_{\mathrm{st}}(\tilde{A})<y$. Letting $y \rightarrow x_{n}+\omega_{s t}\left(C_{0}\right)$, we obtain the following result.

Corollary 3.2. Let $\tilde{A}$ be defined by (3.1). Then $\omega_{\mathrm{st}}(\tilde{A}) \leq \omega_{\mathrm{st}}\left(C_{0}\right)+x_{n}$.

If $C_{0}$ is normal, then $g\left(C_{0}\right)=0$, and with $0^{0}=1$ we have $\zeta(s)=1 / s$ and thus $x_{n}=q$. The following lemma gives us an estimate for $x_{n}$ in the case $g\left(C_{0}\right) \neq 0$.

LEMMA 3.3. Let $q \zeta(1) \leq 1$. Then

$$
x_{n} \leq \sqrt[2 n]{q \zeta(1)}
$$

ProOF. By (3.4), $q \zeta\left(x_{n}\right)=1 \geq q \zeta(1)$. Since $\zeta(s)$ is monotonically decreasing, it follows that $x_{n} \leq 1$. Now (3.5) proves the lemma.

Corollary 3.2 and the Lemma 3.3 yield the following result.

COrollary 3.4. Let $\tilde{A}$ be defined by (3.1) and $q \zeta(1) \leq 1$. Then

$$
\omega_{\mathrm{st}}(\tilde{A}) \leq \omega_{\mathrm{st}}\left(C_{0}\right)+\sqrt[2 n]{q \zeta(1)}
$$

For recent results on the spectra of differential operators see, for instance, the works $[6,7,13,14,15,18,19]$ and the references which are given therein.

\section{Acknowledgement}

I am very grateful to the referee of this paper for many helpful remarks.

\section{References}

[1] W. Arendt, C. J. K. Batty, F. Neubrander and M. Hieber, Laplace Transforms and Cauchy Problems (Springer, Basel, 2011).

[2] W. G. Bade, 'An operational calculus for operators with spectrum in a strip', Pacific J. Math. 3 (1953), 257-290. 
[3] K. Boyadzhiev and R. deLaubenfels, 'Spectral theorem for unbounded strongly continuous groups on a Hilbert space', Proc. Amer. Math. Soc. 120(1) (1994), 127-136.

[4] R. Curtain and H. Zwart, Introduction to Infinite-Dimensional Systems Theory (Springer, New York, 1995).

[5] K.-J. Engel and R. Nagel, A Short Course on Operator Semigroups, Universitext (Springer, New York, 2006).

[6] M. I. Gil', 'Perturbations of operators on tensor products and spectrum localization of matrix differential operators', J. Appl. Funct. Anal. 3(3) (2008), 315-332.

[7] M. I. Gil', 'Resolvent and spectrum of a nonselfadjoint differential operator in a Hilbert space', Ann. Univ. Mariae Curie-Sktodowska Sect. A 66(1) (2012), 25-39.

[8] M. I. Gil', Operator Functions and Operator Equations (World Scientific, Hackensack, NJ, 2018).

[9] M. Haase, 'Spectral properties of operator logarithms', Math. Z. 245(4) (2003), 761-779.

[10] M. Haase, The Functional Calculus for Sectorial Operators, Operator Theory: Advances and Applications, 169 (Birkhauser, Basel, 2006).

[11] M. A. Haase, 'A characterization of group generators on Hilbert spaces and the $H^{\infty}$-calculus', Semigroup Forum 66(2) (2003), 288-304.

[12] T. Kato, Perturbation Theory for Linear Operators (Springer-Verlag, Berlin, 1980).

[13] A. M. Kholkin and F. S. Rofe-Beketov, 'On spectrum of differential operator with block-triangular matrix coefficients', Zh. Mat. Fiz. Anal. Geom. 10(1) (2014), 44-63.

[14] J. Locker, Spectral Theory of Non-Self-Adjoint Two Point Differential Operators, Mathematical Surveys and Monographs, 73 (American Mathematical Society, Providence, RI, 1999).

[15] R. Ma, H. Wang and M. Elsanosi, 'Spectrum of a linear fourth-order differential operator and its applications', Math. Nachr. 286(17-18) (2013), 1805-1819.

[16] C. C. Martinez and A. M. Sanz, The Theory of Fractional Powers of Operators (North-Holland, Amsterdam, 2001).

[17] J. Pruss and H. Sohr, 'On operators with bounded imaginary powers in Banach spaces', Math. Z. 203(3) (1990), 429-452.

[18] A. Zettl and J. Sun, 'Self-adjoint ordinary differential operators and their spectrum', Rocky Mountain J. Math. 45(3) (2015), 763-886.

[19] M. Zhang, J. Sun and J. Ao, 'The discreteness of spectrum for higher-order differential operators in weighted function spaces', Bull. Aust. Math. Soc. 86(3) (2012), 370-376.

MICHAEL GIL', Department of Mathematics,

Ben Gurion University of the Negev, PO Box 653,

Beer-Sheva 84105, Israel

e-mail: gilmi@ bezeqint.net 